\title{
ATMOSPHERIC MOTIONS AND GRANULATION IN COOL STARS - OBSERVATIONS AND THEORY
}

\author{
P.L. COTTRELL \\ Mount John University Observatory \\ Dept of Physics \& Astronomy \\ University of Canterbury, Christchurch, New Zealand
}

\begin{abstract}
.
This review presents a number of aspects in our quest for an understanding of the detailed structure of cool star stellar atmospheres through the study of atmospheric motion. It includes the observational tools (e.g. direct imaging, detailed analyses of line profiles) used to determine the type and amount of motion, as well as the modelling techniques (e.g. simulated images, velocity and pressure fields) used to interpret and describe these motions.

Linkages will be made through the solar-stellar connection to provide insight into the structure of stars where detailed imaging is not possible, and to the determination of the fundamental parameters of stars, such as effective temperature, surface gravity and elemental abundances.
\end{abstract}

\section{Introduction}

As telescopes have been made larger and larger and computers have become more powerful, astronomers and astrophysicists have endeavoured to understand the finer details in the structure of the outer layers of stars through a variety of techniques. So whether one is undertaking a high resolution imaging or spectroscopic observation, or a large scale hydro-dynamical calculation of the motions of a star, one is doing science which is just as fundamental as that which seeks answers to the questions about the large scale structure of the universe. Indeed, as we all know only too well, the basis for our determination of a more precise size of the universe depends critically upon the study of the structure of stars, in particular their mass, size, temperature and energy output. 
In the context of this review I will consider only non-oscillatory atmospheric motion. This is thus referring to the motions due to granulation in the stellar surface layers at the top of the convection zone. The term granulation(s), or granules, is as originally applied by Dawes (1864), where he was endeavouring to introduce a more applicable term for the observations that he made of the solar surface. Older terms included willow-leaves and rice-grains, but Dawes was seeking a description which 'assumes nothing as to exact form or precise character; and (he) venture(d) to hope that the term will be generally adopted.' A reasonable legacy for the Reverend Dawes.

Granules are considered to be the rising gas in the surface layers and have a characteristic dimension of $\mathrm{Mm}$ (millions of metres). They are $\sim 20 \%$ brighter than the material which is moving back down into the star's atmosphere and the timescale for them to rise and disperse is a few minutes. The term 'macro-turbulence' is a term which incorporates granulation and other processes whereby a spectral line is Doppler broadened. A simple visualization of granulation could be considered as a two stream model, one moving upwards and hot and the other downwards and cool, where there is some distribution of rising and falling velocities, which may not be the same, and the granulation velocity goes to zero at the limb of the star.

There have been some links that have been made (Stein \& Nordlund 1991) between the convective motions associated with granulation and the periodic oscillations, but that is beyond the scope of the present review. This review will concentrate on the specific observational and modelling methods that have been and can be developed to obtain a more fundamental understanding of the structure of these stars. This provides input for both stellar interior and stellar atmosphere codes which are needed to deduce the fundamental parameters (radius, temperature, internal structure) of stars.

\section{Observational Tools and Results}

The most obvious technique is to make images of the 'surface' layers. To date, this has only been accomplished for the sun and we await new techniques which will enable other stars to be imaged in a similar way. Nonetheless, it is important that these solar images are combined with a review of this type as only in this way can we provide insights into the type of effects that we are endeavouring to interpret.

There have been many centuries of observations of the solar surface, predating the work of Dawes mentioned above. Many of these earlier works provided an incomplete picture as they were affected by the disturbing motion of the earth's atmosphere. However, with the advent of solar telescopes at sites with stable and excellent seeing (e.g. the Swedish Vacuum 


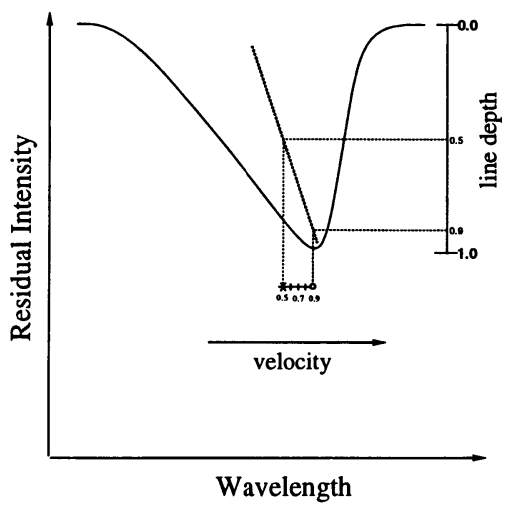

Figure 1. Schematic line bisector for a stellar spectral line. The labeling corresponds to depths in the line profile, where 0.0 is at the continuum and 1.0 is the core of the line (from Wallerstein et al. 1992).

Solar Telescope on La Palma, see Title \& Berger 1996) and the use of (initially) photographic techniques, excellent imaging of the solar disk has shown accomplished and the two major granulation components have been clearly identified. These are the hot rising granules with the cooler falling regions at the margins of these granules.

Given that there is motion in these surface layers spectroscopic techniques can be employed to analyse this motion. Examples of spectroscopic observations of the sun can be found in Collados et al. (1996). To understand what these observations mean requires good instrument calibration, followed by innovative analysis techniques. David Gray provides examples of these techniques (see Gray 1988, particularly Lecture 4).

The tool that is the most useful in this work is to examine the line bisector (see Fig. 1) of a range of spectral lines. For an individual spectral line there is a contribution to the profile from a wide region in the star's atmosphere and when combined with lines of a range of elements, with different excitation and ionisation potential, one has a probe of the outer structure of a star's atmosphere. This includes the temperature and pressure structure and through stellar model atmosphere techniques the effective temperature and surface gravity of the star. The characteristic shape derived from the solar (and some other main sequence stars) observations is a 'C-shape' (see Gray 1988, Fig. 4-9), which includes a blue-shifted and larger contribution from the brighter rising granule and a red-shifted smaller component from the less bright falling intergranular region (Gray 1988, Fig. 4-1). In most other main sequence, and all giant stars, this characteristic shape is a truncated ' $\mathrm{C}$ ', where the lower portion of the ' $\mathrm{C}$ ' is no longer observed (Gray 1988, Fig. 4-25). 
Gray \& Nagel (1989) have combined the bisector data to delineate a 'granulation boundary' in the HR diagram. This boundary runs from F0V to G0Ib. For stars on the cool side of this boundary the line bisectors show the effect of granulation described in the previous paragraph, while on the hot side of the boundary the stars show a very different set of line bisectors, which are not explained by granulation. Gray \& Nagel hypothesised some link between this boundary and deep envelope convective. From discussion with respect to the latest model atmosphere calculations at this Symposium (see Kurucz) this possible link certainly deserves further investigation.

An alternative technique is to just measure the 'mean line shift' for a range of lines of a given species for different excitation potential and line strength (see Glebocki \& Stawikowski 1980). This is a single parameter fit, which is more applicable to situations where the acquisition of detailed line profiles is not possible.

\section{Modelling Techniques}

Gray (1988) used a simple two-stream model to compare with his bisector measurements and although he admits to it being unphysical the model provided insights into the nature of the rising and falling elements in the atmospheres of the sun and stars.

The important hydrodynamic modelling of the surface of the sun has been undertaken by numerous researchers (see Spruit et al. 1990). It relates to both the imaging of the surface layers, with the clear delineation of hot and cool regions, and the motion of these regions. Considerable progress has been made in this area through these models which, although only over relatively small boxes, clearly show the motions which are the cause of the granules.

In the following paragraphs, recent 2D and 3D hydrodynamical calculations of the granulation will be described.

Gadun \& Vorob'yov (1995) have developed 2D hydrodynamic, time dependent models. These use a system of equations for a compressible, radiatively coupled, gravitationally stratified non-magnetic medium the equation of radiative energy transfer using gray Rosseland opacities. Their results cover a $2,000 \mathrm{~km}(\sim 900 \mathrm{~km}$ is the atmosphere) by $4,000 \mathrm{~km}$ region with parameters calculated at $35 \mathrm{~km}$ spacings and at $0.3 \mathrm{~s}$ time steps. Their results with respect to the velocity and temperature profiles (see in particular their Fig. 1) show that they can reproduce the appropriate scales for the variation in distance, time and temperature. For example, granules with a linear dimension of 1,000 to $2,000 \mathrm{~km}$ and several thousand degree contrast in temperature changing over a few minutes seems typical of their calculations. 
Nordlund \& Stein (1991) and Stein \& Nordlund (1991) have produced a series of larger scale, more realistic 3D hydrodynamical calculations. Their results have provided some examples of degraded resolution images which show good similarity to the types of structures observed in solar images (see Fig. 1 of Nordlund \& Stein 1991). Their detailed calculations also provide excellent velocity field and temperature information (see Fig. 3 of Stein \& Nordlund 1991). The velocity field simulations can be compared directly with the solar spectral observations. Because of the 3D work they are also able to give good qualitative agreement between the surface structure and deeper layers (see Fig. 1 of Stein \& Nordlund 1991) and hence a better understanding of the overall structure of the outer layers of stellar envelopes.

These models are obviously only the first steps in a better understanding of the solar atmosphere, which can then be used to map structures in other stars where the imaging data are currently non-existent and the velocity data has much poorer spatial, and generally spectral and time resolution.

\section{Prospects}

The above discussion has highlighted some of the 'tools of the trade' that have been used to understand the detailed structure of stars. There are almost as many questions raised as answered in the observational field as the acquisition of high quality observations is a difficult process and requires very careful calibration of the instrumental system, as well as careful analysis techniques. In the modelling regime, there is a need for more extensive stellar envelope and atmosphere 3D hydrodynamical calculations to compare with the observational data.

However, in spite of the 'well-worn' phrase, "Further observations and models are required", there have been some great successes.

There has been excellent progress in the acquisition of ultra-high spatial and spectral resolution images, as well as polarimetry, of the sun's surface layers. One question raised here is: "What is the smallest scale structure that can be observed and can it be reproduced in the numerical models?"

With the use of very-high spectral resolution of cool stars, what implications are there to make connections with our current understanding of the solar situation? And with temporal coverage and links with other stellar parameters a more complete picture of the interrelation between the different observations (e.g., magnetic effects, luminosity variations - see Gray et al. 1996).

Considerable progress is being made in our understanding of the effects of motions on the determination of the temperature structure of cool stars, the effective surface gravity of these stars and the effect that atmospheric motion has on the determination of the abundances derived for these cool 
stars. Under- and over-excitation and ionisation effects will also be better understood with a more complete picture of the structure of the outer layers of these atmospheres.

The next generation of observations and models will surely provide some further progress in this area and enable astronomers to make significant advances in this area of stellar astrophysics.

\section{References}

Collados, M., Rodriguez Hidalgo, I., Ballesteros, E., Ruiz Cobo, B., Sanchez Almeida, J. \& del Toro Iniesta, J.C. 1996 A\&A Supp., 115, 367-77.

Dawes, W.R. (1964) MNRAS, 24, 161.

Gadun, A.S. \& Vorob'yov, Yu.Yu. (1995) Solar Physics, 159, 45-51.

Glebocki, R. \& Stawikowski, A. (1980) in Stellar Turbulence, (eds D.F. Gray \& J.L. Linsky) IAU Coll. 51, p55.

Gray, D.F. (1988) in Lectures on spectral-line analysis: $F, G$ and $K$ stars, Lecture 4 Stellar Granulation.

Gray, D.F. \& Nagel, T. (1989) ApJ, 341, 421-6.

Gray, D.F., Baliunas, S.L., Lockwood, G.W. \& Skiff, B.A. (1996) ApJ, 465, 945-50.

Nordlund, $\AA$. \& Stein, R.F. (1991) in 'Challenges to theories of the structure of moderatemass stars' (eds D. Gough \& J Toomre) Lecture Notes in Physics, 388, 141.

Paxman, R.G., Seldin, J.H., Lofdahl, M.G., Scharmer, G.B. \& Keller, C.U. (1996) ApJ, 466, 1087-99.

Spruit, H.C., Nordlund, Å. \& Title, A.M. (1990) Ann. Rev. Astron. Astrophys., 28, 263.

Stein, R.F. \& Nordlund, $\AA(1991)$ in 'Challenges to theories of the structure of moderatemass stars' (eds D. Gough \& J Toomre) Lecture Notes in Physics, 388, 195.

Title, A.M. \& Berger, T.E. (1996) ApJ, 463, 797-807.

Wallerstein, G., Jacobsen, T.S., Cottrell, P.L., Clark, M. \& Albrow, M.D. (1992) MNRAS, 259, 474-88.

\section{DISCUSSION}

O'Mara: In your work have you looked at the hill vortex model developed by Roger Ulrich?

Cottrell: No. Rather than looking at individual models for convection, I was seeking full hydrodynamical descriptions of the convection/granulation problem.

Bell: When interpreting very accurate radial velocity observations of Cepheids, which show different velocities for different elements, should we consider that the Gray and Nagel effect is simply superimposed on the Cepheid oscillation?

Cottrell: I would suggest that one is going to require full hydrodynamical non-LTE models (incorporating the stellar envelope and atmosphere) to interpret the line level effects that are observed in Cepheid stars. Using any form of 'add-on' effect would not be a very satisfactory approach. 